\title{
The Yin and Yang of Integrated Care: Systemic Imperatives for Traumatic Brain Injuries
}

\author{
Denis H.J. Caro*
}

Full Professor, Telfer School of Management, University of Ottawa, Ottawa, Canada

\begin{abstract}
Traumatic brain injuries remain a $21^{\text {st }}$ century scourge that remains subterranean and mostly invisible in our societies. Integrated care systems for traumatic brain injury patients effectively integrate the key systemic components horizontally and vertically so as to improve patient outcomes and systemic performance. This review paper briefly identifies the nature of traumatic brain injuries in Canada and the European Union. Internationally, there is a wide spectrum of health care systems and supporting infrastructures that reflect a very diverse and complex range of societal values, political ideologies, and states of economic and social development. As a consequence, each nation or political entity will have a different response or ability to respond to traumatic brain injuries. The manuscript underscores the need to recognize traumatic brain injuries as a universal public health challenge and briefly identifies the systemic components of care in Canada and the European Union that are sociopolitical entities that have a blend of both government-directed and marketeconomies. The paper posits that there are four evolving systemic imperatives that should be considered in providing effective care to the traumatic brain injury patients for the $21^{\text {st }}$ century. These include: integrated care planning; integrated information systems; virtualized continuity of care and health leadership imperatives. How each socioeconomic entity integrates these building blocks will vary, as will the effectiveness to these types of injuries. The paper concludes with a brief discussion of public health choices that lie at the heart of the scourge of traumatic brain injuries.
\end{abstract}

Keywords: Health leadership, integrated care systems, regional health authorities, traumatic brain injuries.

\section{INTRODUCTION}

Over the last 15 years, there has been a significant thrust towards integrated care systems internationally [1-4]. The need for relational and organizational continuity of care and collaboration of care professionals and stakeholders has been underscored through the literature [5-7]. The mission of integrated care systems is to create health and social ecologies for the provision and delivery meaningful, timely and contiguous health and social care effectively. Such is the case with traumatic brain injuries which remains a subterranean $21^{\text {st }}$ century scourge and mostly invisible in our societies [8]. Indeed, traumatic brain injuries constitute "yin" forces, or a destructive presence, in our social ecology. Public values and choices and the inherent risks of life have contributed to this international population health challenge with dire implications for health costs and quality of life to its victims. Traumatic brain injury care systems are those, which efficiently and effectively integrate all of the key care pathways horizontally and vertically across the system. The fundamental mission of such care systems is to reduce mortality and morbidity rates, risks and incalculable human suffering from neuro-traumatic events. These systems in effect are the "yang", or positive forces, responding to and counterbalancing the "yin" or negative scourge of traumatic brain injuries [9]. Ultimately, these systems seek to promote primary and secondary

\footnotetext{
*Address correspondence to this author at the Telfer School of Management, University of Ottawa/ Université d'Ottawa, 55 Laurier East, Ottawa, Ontario, K1N 6N5, Canada; Tel: + (613) 562-5800, Ext: 4742;

Fax:+(613)+562-5164; E-mail: drcaro@uottawa.ca
}

prevention strategies and assure positive care outcomes, full physical and cognitive functionality and social reintegration. Paradoxically, these care systems remain largely in a state of "discontinuity" operating largely in silos, each bounded with separate data, information, applications and technological architectures. This paper underscores possible directions for $21^{\text {st }}$ century evolution for integrated care systems through the challenges of care for persons with traumatic brain injuries. Metaphorically, neither the "yin nor yang" forces are yet in balance. Only an integrated holistic management approach can bring the presence of these two in harmony.

Internationally, there is a wide spectrum of health care systems and supporting infrastructures that reflect a very diverse and complex range of societal values, political ideologies, and states of economic and social development. These include largely market-oriented economies, such as the United States, to largely government- directed economies, such as the People's Republic of China. This manuscript focuses on those sociopolitical entities, such as Canada and the European Union, that have a blend of both government -directed and market-economies. It is also recognized the international war zones have quite different challenges in dealing with the scourge of traumatic brain injuries. As a consequence, each socioeconomic or sociopolitical entity will have a different response or ability to respond to traumatic brain injuries. This manuscript does not pretend to espouse one approach or solution fits all. Yet the manuscript underscores the need to recognize traumatic brain injuries as a universal public health challenge and posits that there are fundamental building blocks that should be considered in effectively responding to the challenges. How each nation or 
political entity integrates the building blocks will vary as will the effectiveness to this universal scourge.

\section{THE YIN OF TRAUMATIC BRAIN INJURIES}

Traumatic brain injuries are externally-inflicted assaults that are the most common global causes of death and disability in people under 45 years of age and they pose major health and socioeconomic challenges, which affect all societies internationally [10]. In Canada, traumatic brain injuries are a leading cause of permanent disability and result from falls $(28 \%)$, motor vehicle accidents $(20 \%)$, contact sports (19\%) and violent assaults and attempted suicides (11\%) $[11,12]$. These injuries particularly afflict adolescents and young adults and over 600,000 Canadians and 8,600,000 Europeans continue to live with the long term consequences. The annual health care costs and life years lost due to death and disabilities from these injuries amounted to over an estimated \$7 billion CDN in Canada and 94 billion euros in the European Union in 2012 [13]. Blast trauma causes over 35 per cent of all traumatic brain injuries and are deemed as "signature wounds" in war zones and pose added significant clinical challenges [14-18].

The literature is replete with the clinical implications of traumatic brain injuries $[19,20]$. There are three major clinical parameters to classify the degree of severity of traumatic brain injuries as either minor, moderate or severe. This includes 1.the length of time of Loss of Consciousness (LOC), or Alteration of Consciousness (AOC); 2. the length of time of Post-Traumatic Amnesia (PTA); and 3.the Glasgow Coma Score (GCS), a universally recognized neurological functional assessment tool [10]. Minor traumatic brain injuries typically constitute 60 percent of cases and pose clinical challenges of accurate identification and under-diagnosis [21-23].

Table 1 summarizes the clinical features for each severity category; the estimated percentage of traumatic brain injury cases, according to the clinical literature and an estimation of the annual number of cases in Canada and the European Union, based on the 2012 populations [11]. These numbers reflect areas of the world that are have developed and stable economies and are not active war conflict zones. More precise data internationally would be very difficult given the relatively underdeveloped clinical databases.

Traumatic brain injuries, be they minor, moderate or severe, create significant clinical and rehabilitation challenges for a wide spectrum of care professionals, including critical care nurses, emergency physicians, family practitioners, neurologists, neurosurgeons, paramedics, psychologists, psychiatrists, occupational and physical therapists, social workers, as well as family caregivers [24-27]. Delays in diagnosis and treatment complicate the prognosis for these patients, 35 percent of whom also present with poly-traumatic manifestations. About 15 percent of minor traumatic brain injury patients experience chronic and persistent symptoms for more than one year [21-23]. For the 10 percent who sustain moderate brain injuries, chronicity can be life-long [28]. Severe traumatic brain injuries afflict about 30 per cent of the total brain injury population and carry a 90 per cent mortality rate [29]. For those who have sustained blast trauma in militarized or violent zones, the long-term societal impacts of permanent brain injuries are even more significant $[15,16$, 30]. For brain injury survivors, the physiological and psychological consequences have a deleterious impact on the psycho-social integrity and functioning. Cognitive recovery is clinically complex, individualistic and unpredictable, making such injuries a silent and costly epidemic. For example, post-traumatic stress disorders affect over 40 per cent of the afflicted population with significant consequences for social re-integration and increased risks of substance abuse, homelessness, vulnerability and susceptibility to violence [31-33].

\section{THE YANG OF TRAUMATIC BRAIN INJURIES: SYSTEMIC RESPONSES}

The goal of integrated care systems for traumatic brain injury patients is to optimize cognitive, functional and therapeutic outcomes for those so afflicted. In essence, it is to sustain life and recover quality of life in terms of physical, mental, social and spiritual well-being. These systems require cross-functional and inter-organizational effective coordination and management across seven subsystems, each with its unique clinical and systemic management challenges. These include:

- Field emergency care

- Transportation care

- Emergency unit care

- Neuro-trauma center care

- Psychiatric care

- Rehabilitative care

- Social care [11]

Table 1. TBI severity parameters and assessment tools.

\begin{tabular}{|c|c|c|c|c|c|}
\hline Severity of TBI & $\begin{array}{c}\text { Loss /or alteration } \\
\text { of consciousness } \\
\text { (LOC/AOL) }\end{array}$ & $\begin{array}{c}\text { Post Trauma } \\
\text { Amnesia (PTA) }\end{array}$ & $\begin{array}{c}\text { Glasgow Coma } \\
\text { Score (GCS) }\end{array}$ & $\begin{array}{c}\text { Trauma Brain } \\
\text { Injury (TBI) } \\
\text { Cases (\%) }\end{array}$ & $\begin{array}{c}\text { Estimated Annual Cases of } \\
\text { Traumatic Brain Injuries (TBI) } \\
\text { in Canada and the } \\
\text { European Union in 2012 }\end{array}$ \\
\hline \hline Minor & $<30$ minutes & 24 hours & $13-15$ & $60.0 \%$ & $1,770,000$ \\
\hline Moderate & $>30$ minutes & $1-6$ days & $9-12$ & $19.0 \%$ & 560,500 \\
\hline Severe & 24 hours & 7 days & $3-8$ & $21.0 \%$ & 619,500 \\
\hline TOTAL & & & & $100.0 \%$ & $2,950,000$ \\
\hline
\end{tabular}




\section{- Field Emergency Care}

Paramedics with advanced trauma life support skill sets and competencies assess suspected brain injuries cases and prevent neuro-worsening. Field detection and screening of brain injury manifestations through the use of established clinical algorithms and guidelines remain crucial $[10,11]$.

\section{- Transportation Care}

Optimal recovery from traumatic care injuries depend on rapid assessment, early and efficient clinical intervention, safe and effective evacuation to specialized neuro-trauma centers that provide diagnostic and neurosurgical care. Patients with such injuries pose logistical and transportation care management challenges. Stable transportation of patients and multidisciplinary care coordination of critical care transportation teams of critical care paramedics, emergency physicians, intensivists and critical care nurses is essential [10].

\section{- Emergency Unit Care}

Further screening, triage and stabilization occur at emergency care units at base hospitals that usually have access to CAT and MRI scanners and more advanced diagnostic and treatment resources. This is important in the clinical attempts to pre-empt adverse prognostic outcomes. Moderate and severe cases often require greater panoply of care resources, skill sets and competencies. In these cases, base hospitals serve as critical care holding stations to stabilize the clinical picture of patients before evacuation to neuro-trauma care units [10].

\section{- Neurotrauma Center Care}

The transfer of moderate and severe trauma brain injury patients to neuro-trauma centres is paramount for survival and improved therapeutic outcomes. Such centers offer greater access more advanced neuroimaging tools and care resources that prevent and limit progressive brain damage. These centers also have polyvalent trauma services to attend to complex trauma cases $[34,35]$.

\section{- Rehabilitative Care}

The follow-up of traumatic brain injury patients is critical, as the consequences are substantial, long-term and affect the quality of life [36]. Such patients face diverse outcomes, including complete recovery and independence, moderate or severe disability, long-term comatose or vegetative states, or death. Early and intensive rehabilitation is important in achieving the best possible functional and therapeutic outcomes for patients. Effective coordination of services, such as cognitive rehabilitation and remediation, occupational therapy, optometry, physical therapy, and pharmacological therapies minimize the risk of subsequent medical complications, improve patient satisfaction and maximize functional recovery [37].

\section{- Psychiatric Care}

Survivors of traumatic brain injuries represent a very vulnerable population, that suffer from diverse psychiatric sequelae, such as bipolar disorders, clinical depression, generalized anxiety disorders, post-traumatic stress disorders, suicide ideation and substance abuse [38]. Suicidality is also an important problem [39]. Early psychological and psychiatric intervention and care coordination of mental health care providers is essential. Such care includes clinical management, psychological counselling and pharmacologic treatment and in some cases, residential care.

\section{- Social Care}

Survivors of traumatic brain injuries invariably face challenges in readjusting to family and work life, interpersonal relationships and social functioning. Social care strategies seek to maximize individual independence and reintegration into stable family units and work environments [40]. Effective caregiving, home care, social support and respite services are essential in the face of complex processes of adapting to, coping with and surmounting the myriad of physical, cognitive and behavioural challenges that complicate recovery. Stable care resources, emotional and financial support are essential for the patients and their families [11].

\section{REBALANCING YIN AND YANG FORCES: SYS- TEMIC IMPERATIVES OF INTEGRATED CARE}

Integrated care for traumatic brain injury patients need to focus on the delivery systems, performance management and outcome based learning in the $21^{\text {st }}$ century [4]. There are four systemic imperatives, or building blocks, for such more effective integrated care systems.

\section{- Effective Integrated Care Planning}

Continuity of care from the field emergency to social care is most important to positive outcomes for patients with traumatic brain injuries. It is what others have underscored as relational continuity [7]. Discontinuity of care occurs at the transition points where patients transfer from one subsystem to another. Each transition entails a transfer of professional care accountabilities and information. Management continuity in the form of clear clinical and management policies and procedures across these subsystems remains important. Case coordinators are essential to help patients and their families navigate through complex care systems from emergency care to social care. Fully accountable care coordinators need to work with integrated multidisciplinary care providers to assure management and relational continuity and the best care and outcomes for these patients. Regional health authorities remain the cogent foundation for horizontal and vertical integration for care components for traumatic brain injury patients. These bodies form the nexus of management and relational continuities for traumatic brain injury patients, as well as the basis for systemic performance monitoring and outcomes learning and sharing. In Canada, the ultimate accountability for quality care services still remains largely at the care facility level. Regional health organizations have coordinating roles across health institutions, but have limited real legislative authority to integrate care networks to assure the best therapeutic and functional outcomes for patients with traumatic brain injuries. Stable financial and resource support from governance bodies remains a challenge. Moreover, there is a manifest need for more cooperation from individual institutions that currently continue to jealously guard their own independence and organizational survival as primordial. 


\section{- Effective Integrated Information Systems}

Integrated information infrastructures form the basis for effective flow of information across care subsystems for traumatic brain injury patients. They are the basis for effective communication, performance monitoring, systemic improvements for optimal outcomes for patients with traumatic brain injuries. These infrastructures incorporate data warehouses and data mining technologies and a vast array of software applications from business intelligence systems to integrated patient databases. Moreover, the deployment of expert systems, nanotechnology and neural network technologies and the increasing availability of clouding computing services potentiate the integration of information flows through care systems in ways unimaginable even ten years ago. Regional health authorities remain the most cost-effective and logical hub for the information flow transfers and infrastructures. The development of these infrastructures require extensive dialogue, communication and collaboration of diverse stakeholders, ranging from, paramedical and emergency services, medical transportation units, base hospitals, neurotrauma care centers, neuro-trauma care centers, social care agencies, professional associations and care providers across service communities. Moreover, the private sector in hightechnology offers unique and cost-effective options to support the informational continuity throughout the system [7]. Effective engagement depends on proactive strategic partnerships with governance bodies and health care organizations $[7,41,42]$. Trust relationships form the key prerequisite to engagement and collaboration across sectors and care providers across the entire care system. This too underscores the importance of a bottoms-up approach to the evolution of integrated care systems for traumatic brain injury patients.

\section{- Virtualized Continuity of Care Systems}

War theatres and the ensuing traumatic brain injuries have spawned the drive for more effective tele-medical applications that support neurological assessment, neurosurgical treatment, occupational and physical rehabilitation, psychiatric intervention and behavioral therapies [43, 44]. The future for integrated care systems is one of virtualized care that brings brain injury care to patients. Neuro-medical care for brain trauma patients will increasingly move to the field. Indeed, the future includes cloud coordination of telediagnosis, robotic neurosurgery, tele-monitoring, neuroconsultations, tele-rehabilitation and tele-home care, regardless of spatial and time constraints. Tele-medical applications for traumatic brain injury patients are part of the evolving future e-health care systems, including surgical robotics and thought-controlled prosthetics. Handheld and weartechnologies with screening algorithms and neurocognitive test batteries will link to patient history. Home tele-health expands the reach of visiting nurses, who can monitor their patients remotely through monitoring devices. Moreover, the $21^{\text {st }}$ century also holds promise for neuro-protective technologies and neurobiology advances including the regeneration of neurological functions, molecular and gene therapy, pharmacology therapies that could mitigate the impact of traumatic brain injuries and its deleterious sequelae, such as posttraumatic stress disorder [32, 45]. Regional health bodies have the potential to provide the forum for dialogue, innovation, collaboration and implementation of virtualized conti- nuity of care for traumatic brain injury patients across the community.

Surgical robotics, tele-home care and tele-rehabilitation and other technologies are on the e-health horizon in many nations. The deployment of such technologies will evolve to become part of the public health scene and dialogue in the $21^{\text {st }}$ century although at this juncture, it is too early to assess their clinical beneficial effects. They may be one way to manage the long-term costs and declining health resources in some nations. The wisdom with which these technologies are effectively deployed will determine their efficacy in leveraging the quality of care and quality of life of victims of traumatic brain injuries.

\section{- Transformational Health Leadership and Vision}

The responsibility for the evolution of integrated care systems rests with leadership [46]. Proactive vision and strategic leadership unlocks the power of innovative technologies to transform care systems for traumatic brain injury patients. It remains for regional, national and international governance organizations to provide and support proactive leadership with essential resources to foster the integration of care systems for traumatic brain injury patients. Strategic direction, dialogues and collaborative partnerships through transformational leadership are needed. Stakeholder consultations through a bottom-up and evolutionary approach are needed in integrated care of traumatic brain injury patients. Transformational leadership is the essential spark and ingredient to make this happen. This requires substantial strategic planning and judicious change management strategies to have diverse care professionals across the community of traumatic brain injury care to communicate and understand each other. Regional and national bodies should be the orchestrators and harmonizers of the bottom- up approach with full care provider engagement. Value systems across professional domains need to be mutually understood and converge in the interests of relational continuity for patients.

\section{BEYOND PARADIGMS OF INTEGRATED CARE}

Traumatic brain injuries continue to pose serious public health challenges internationally. The current modalities of care pathways for such patients will be increasingly unsustainable on the face of growing health care demands on so many fronts. The evolution towards integrated care systems for traumatic brain injuries requires transformational leadership, vision and commitment. That vision needs to go well beyond primary prevention strategies, community education and integrated care systems. It requires the evolution of new international community values and norms. In the dawn of the $21^{\text {st }}$ century, the world remains a dangerous place with innumerable vulnerabilities and threats. Too many are the societies and cultures that are subject to human destruction and violence, through policies that tolerate, if not condone, the proliferation of firearms and weaponry, destructive behaviours, alcohol and substance abuse, violent sports, fratricidal conflicts and unsafe transportation systems. The "yin" forces in effect continue to dominate the societal landscape. Integrated care systems for traumatic brain injury patients serve as safety nets for essentially violence-tolerant societies. Within Canadian social norms, prevention focuses on public 
education, safety awareness and the use of neuro-protection devices, as deemed socially acceptable.

Cogent primary prevention and health promotion strategies and zero-tolerance policies for destructive and risky behaviour are seen as draconian and are not part of societal dicta, or visions. Canadian culture does not yet universally regard health and well-being as a critical value, a right of every human being and as a worthy investment in people. Health care remains associated with sickness care and financial drains with interminable costs and expenditures. A good health promulgates socio-economic integrity and viability of human ecology is not yet universally recognized. Economic and vested interests in violent and militarized cultures remain part of the socio-political landscape may not change for generations to come. Socio-cultural transformations that would root out the causes of traumatic care injuries will be inexorably slow. In the interim, integrated care systems will continue to be an essential goal to reduce the deleterious impacts of such abominable injuries- the ever-present yin forces. Until societies embrace values of humanitarianism, non-violence, peace and health as the foundation of physical, mental, social and spiritual well-being, integrated care systems will remain paramount in confronting the international scourge of traumatic brain injuries.

Given the diversity of nations and political entities across the planet, it remains to each to configure the building blocks of integrated care of traumatic brain injuries in ways most congruent to individual sociopolitical and socioeconomic contexts and realities. If mitigating the scourge of traumatic brain injuries and to manifest human suffering and consequences of this scourge is the central goal, then the building blocks or systemic imperatives should be considered. Effective response to the scourge of traumatic brain injuries should ideally be comprehensive and integrated, as should it be to the myriad of other public health challenges. Such injuries not only manifest clinically, but have socio-economic implications well beyond a strictly medical arena. Whatever building blocks are absent will translate into socioeconomic costs that each political entity must be willing to bear. For many nations, the cost and human consequences of the status quo may not be sustainable in the long-term. Such injuries mandate integrated visions and strategic responses that are the very essence of international public health endeavors.

\section{CONFLICT OF INTEREST}

The author confirms that this article content has no conflict of interest.

\section{ACKNOWLEDGEMENTS}

This paper is based on the ideas and conceptions of Dr. Denis Caro at the Telfer School of Management at the University of Ottawa/ l'Universite d'Ottawa. It is inspired by the courageous suffering of victims of traumatic brain injuries and to all health professionals dedicated to the alleviation of their suffering.

\section{REFERENCES}

Ahgren B. Competition and integration in Swedish health care. Health Pol 2010; 96(2): 91-7.
[2] Glasby J. Key lessons from integrated care in Europe. Int J Integra Care [serial online], North America, 12, Jan. 2012. Available at: http://www.ijic.org/index.php/ijic/article/view/830/1518 [Date accessed: 19 Mar. 2013].

[3] Minkman M. Developing integrated care: Towards a development model for integrated care. Int J Integra Care [serial online], North America, 12, Oct. 2012. Available at: http://www.ijic.org/ index.php/ijic/article/view/1060/1907 [Date accessed: 19 Mar. 2013].

[4] Schrijvers G. The integration of chronic care and emergency medicine. Int J Integra Care [serial online], North America, 8, Jun. 2008. Available at: http://www.ijic.org/index.php/ijic/article/view/ 233/465 [Date accessed: 21 Mar. 2013].

[5] Ahgren B. Axelsson R. Determinants of integrated health care development: chains of care in Sweden. Int J Health Plan Man 2007; 2:145-57.

[6] Bodenheimer T. Coordinating care: a perilous journey through the health care system. New Eng J Med 2008; 358(10):1064-71.

[7] Caro D. The axis and nexus of e-health alliances in 2020: A commentary. Can J Pub Health 2005; 96(4): 291-3.

[8] Vaishnavi S, Rao V, Fann J. Neuropsychiatric problems after traumatic brain injury: Unraveling the silent epidemic. Psychosomatics 2009; 50(3): 198-205.

[9] De Mente B. The Chinese have a word for it: the complete guide to Chinese thought and culture. Chicago, USA: Passport books; 2000. pp. 448-9.

[10] Imhof HG. Lenzlinger PM. Management of traumatic brain injury: Application of guidelines for diagnostics and therapy. Eur $\mathrm{J}$ Trauma 2005; 31(4): 331-43.

[11] Caro D. Traumatic brain injury care systems: 2020 transformational changes. Glob J Health Sci 2011; 3(1): 19-29.

[12] Park E, Bell J, Baker A. Traumatic brain injury: Can the consequences be stopped? Can Med Assoc J 2008; 178(9): 1163-70.

[13] Caro D. Towards systemic sustainable performance of traumatic brain injury care systems: Emergency leadership frontiers. Int $\mathrm{J}$ Emerg Med 2010; 3(4): 357-65.

[14] Almogy G, Rivkind AI. Terror in the 21st century: Milestones and prospects-Part I. Cur Problems Surg 2007; 44: 496-554.

[15] Bhattacharjee Y. Shell shock revisited: solving the puzzle of blast trauma. Science 2008; 319:406-8.

[16] Hayward P. Traumatic brain injury: the signature of modern conflicts. Lancet Neurol 2008; 7(3): 200-1.

[17] Snell F, Halter M. A signature wound of war: Mild traumatic brain injury. J Psychosoc Nur Ment Health Serv 2010; 48(2): 22-8.

[18] Wolf S, Bebarta V, Bonnett C, et al. Blast injuries. Lancet 2009; 374(9687): 405-15.

[19] Hulme J. Resuscitation of patients after traumatic brain injury. Trauma 2008; 10(1): 55-63.

[20] Mathias JL, Wheaton P. Changes in attention and informationprocessing speed following severe traumatic brain injury: A metaanalytic review. Neuropsychology 2007; 21:212-23.

[21] Bryant R. Disentangling mild traumatic brain injury and stress reactions. Eng J Med 2008; 358(5): 525-7.

[22] Petchprapai N, Winkelman C. Mild traumatic brain injury: Determinants and subsequent quality of life. A review of the literature. J Neurosci Nur 2007; 39(5): 260-72.

[23] Ruff R. Two decades of advances in understanding of mild traumatic brain injury. J Head Trauma Rehab 2005; 20(1): 5-18.

[24] Von Wild, K. Neurorehabilitation following craniocerebral trauma. Eur J Trauma 2005; 31(4): 344-58.

[25] Brooks JR. Veterans' health system blazing trails. Can Med Assoc J 2008; 179(6): 518-9.

[26] Lux W. A neuropsychiatric perspective on traumatic brain injury. J Rehab Res Develop 2007; 44(7): 951-62.

[27] Phipps S, Richardson P. Occupational therapy outcomes for clients with traumatic brain injury and stroke using the Canadian occupational performance measure. Am J Occup Therapy 2007; 61(3): 328-34.

[28] Maas A, Stocchetti N. Bullock R. Moderate and severe traumatic brain injury in adults. Lancet Neurol 2008; 7(8): 728-41.

[29] Winslade W. Severe brain injury: Recognizing the limits of treatment and exploring the frontiers of research. Camb Quart Healthcare Eth 2007; 16(2): 161-8.

[30] Kocsis J, Tessler A. Pathology of blast-related brain injury. J Rehab Res Develop 2009; 46(6): 667-72. 
[31] Bigler E. Neuropsychology and clinical neuroscience of persistent post-concussive syndrome. J Int Neuropsychol Sociol 2008; 14:122.

[32] Keane T. Posttraumatic stress disorder: Future directions in science and practice. J Rehab Res Develop 2008; 45(3): 7-9.

[33] Stein M, McAllister T. Exploring the convergence of posttraumatic stress disorder and mild traumatic brain injury. Am J Psych 2009; 166(7): 768-76.

[34] MacKenzie EJ, Rivara FP, Jurkovich GJ, et al. A national evaluation of the effect of trauma-center care on mortality. New Eng J Med 2006; 354: 366-78.

[35] Blum N, Fee E. Critical shortcomings at Walter Reed Army Medical Center create doubt. Am J Public Health 2008; 98(12): 215960

[36] Mathias JL, Wheaton P. Changes in attention and informationprocessing speed following severe traumatic brain injury: A metaanalytic review. Neuropsychology 2007; 21: 212-23.

[37] Gutman S. Special issue on brain injury: Traumatic brain injury and stroke. Am J Occup Therapy 2009; 63(5): 523-4

[38] Simpson G, Tate R. Preventing suicide after traumatic brain injury: implications for general practice. Med J Aus 2007; 187(4): 229-32.
[39] Simpson G, Tate R. Suicidality in people surviving a traumatic brain injury: prevalence, risk factors, and implications for clinical management. Brain Inj 2007; 21:1335-51.

[40] Williamson J. The disarmament, demobilization and reintegration of child soldiers: Social and psychological transformation in Sierra Leone. Intervention: Psychosocial work and counselling in areas of armed conflict. Int J Mental Health 2006; 4:185-205.

[41] Ahgren B. Mutualism and antagonism within organisations of integrated health care. J Health Organ Manage 2010; 24(2): 396411.

[42] Caro D. Forging e-health partnerships: Strategic perspectives for international executives". Health Care Manage Rev 2005; 30(2) 174-81.

[43] Caro D. Deconstructing symbiotic dyadic e-health networks: Transnational and transgenic perspectives. Inte $\mathrm{J}$ Info Manage 2008; 28(2): 94-101.

[44] Girard P. Military and VA telemedicine systems for patients with traumatic brain injury. J Rehab Res Develop 2007; 44(7): 1017-26.

[45] Keltner NL, Cooke B. Traumatic brain injury-war related. Perspect Psychiatric Care 2007; 43(4): 223-6.

[46] Caro D. Towards traumatic brain injury care systems: Health leadership imperatives in Canada. Healthcare Manage Forum 2011; 24(1): 25-30.

(C) Denis H.J. Caro; Licensee Bentham Open.

This is an open access article licensed under the terms of the Creative Commons Attribution Non-Commercial License (http://creativecommons.org/licenses/by-nc/3.0/) which permits unrestricted, non-commercial use, distribution and reproduction in any medium, provided the work is properly cited. 